\title{
Improved field emission via laser processing of carbon nanotubes on paper substrates
}

\author{
S. M. Lyth ${ }^{a}$, S. J. Henley, and S. R. P. Silva \\ Nanoelectronics Centre, Advanced Technology Institute, University of Surrey, Guildford, Surrey \\ GU2 7XH, United Kingdom
}

(Received 14 October 2008; accepted 30 March 2009; published online 24 April 2009)

Field emission (FE) is the emission of electrons from a solid by the application of a high electric field via quantum mechanical tunneling of electrons through a potential barrier. Carbon nanotubes (CNTs) have excellent FE properties, ${ }^{1,2,3,4}$ owing to their large aspect ratio and high electrical conductivity, ${ }^{5}$ and they have been extensively investigated as cold cathodes for lighting and flat panel displays. ${ }^{6,7,8}$ The realization of low cost, large area CNT cathodes is crucial if FE devices are to become contenders in the marketplace. Several groups have experimented with field emitting inks and ink-jet printing of CNTs for electronics applications. ${ }^{9,10,11,12}$ Ink-jet printing, dip coating, screen printing, and processing on flexible substrates all lend themselves to industrialization and continuous manufacturing processes with clear cost benefits over vacuum deposition, thermal evaporation, and/or chemical vapor deposition. Recently, acid-oxidized CNT inks have been printed using ink-jet technology and their electrical properties were investigated. ${ }^{13,14}$

The morphology of a surface plays a crucial role in determining its FE properties. ${ }^{15,16}$ The local field at the emission site governs FE, so micron and nanoscale protrusions can enhance FE due to increased electric field concentration at their surfaces. This enhancement is described by the field enhancement factor $(\beta)$; the factor by which the local electric field exceeds the macroscopic applied electric field. Therefore, by altering the morphology of an emitter, $\beta$ and the FE properties can be modified. In this article the deposition of multiwall carbon nanotube ink (MWNT) onto paper substrates via a straightforward dip-coating technique is investigated as a means to increase $\beta$ by taking advantage of the inherent roughness of the substrate. Paper substrates were chosen as low cost, flexible, readily available materials, and as established substrates for printing technologies. It has already been determined that MWNTs deposited onto paper substrates can have excellent FE properties, which can be tailored according to the morphology of the specific paper substrate utillzed. ${ }^{17}$ Here, the effects of a postdeposition excimer laser treatment on the morphology and, therefore, FE characteristics of such samples are investigated.

MWNTs with a nominal diameter of $10 \mathrm{~nm}$ were purchased from Nanocyl and ultrasonically dispersed in a 3:1 concentrated sulfuric and nitric acid mixture for $10 \mathrm{~min}$. The mixture was refluxed at elevated temperature for 40 min before diluting with de-ionized water. This process results in the formation of carboxylic acid groups attached to the MWNT ends and sidewalls, which interact with water molecules via hydrogen bonding. Large particles and agglomerates were removed from the solution by centrifuging. The centrifuged liquid was carefully decanted, filtered, and washed with de-ionized 
water until a slurry withpH 6-7 was obtained. This was added to a small amount of de-ionized water and sonicated to redisperse the MWNTs. The resulting product was a stable dispersion of acidoxidized MWNTs in water. Acid treatment also serves to purify MWNTs removing catalytic particles and amorphous carbon. For the sake of clarity, acid-oxidized MWNTs are herein referred to as oMWNTs. The o-MWNT loading $(3.5 \mathrm{mg} / \mathrm{ml})$ was determined by drying and weighing a portion of the oMWNT ink.

Commercially available laboratory filter paper was chosen as a substrate, specifically for its complex micron-scale structure. Samples were fabricated by drop casting the o-MWNT solution onto the paper substrates and then baking for $10 \mathrm{~min}$ at $100^{\circ} \mathrm{C}$ to remove moisture. Subsequently, the sample was exposed to single $25 \mathrm{~ns}$ pulses from a $248 \mathrm{~nm}$ UV excimer laser (Lambda Physik LPX210i) over a range of energies. The area of the laser spot was $0.18 \mathrm{~cm}^{2}$, which was used to calculate the incident fluence on the sample surface. Large areas can be treated by translating the sample with respect to the laser. Irradiation was carried out at a vacuum better than $10^{-4}$ Torr.

Figures 1a,1b,1c,1d,1e,1f show scanning electron microscope (SEM) images of o-MWNTs on paper substrates, irradiated with laser fluences of $0,28,56,83,111$, and $139 \mathrm{~mJ} / \mathrm{cm}^{2}$, respectively. In Figs.1a,1b, the paper is homogeneously coated with an o-MWNT layer and no change in the surface is observed after laser irradiation at $28 \mathrm{~mJ} / \mathrm{cm}^{2}$. It is concluded that this laser fluence has insufficient energy to affect the o-MWNT layer. In Figs. 1c,1d,1e, portions of the o-MWNT layer have peeled away from the underlying paper substrate and protrude from the surface almost perpendicularly. These surface features are just a few tens of nanometer thick and tens of microns in length. The uniformity of this surface modification extends over hundreds of microns across the substrate and seems to be limited only by the Gaussian profile of the laser spot. The degree of surface modification increases as the laser fluence increases. Finally, in Fig. $1 f$ a larger degree of damage to the o-MWNT layer is observed. Some charging effects are observed toward the bottom of the image, where the oMWNT layer has been almost completely removed and the underlying insulating paper substrate is exposed to the electron beam. At these high fluences, it is possible that the extensive damage to the sample is due to the decomposition of the o-MWNTs caused by C-C bond breaking. ${ }^{18,19,20}$ The remaining protruding o-MWNTs form much smaller surface features, comprising clusters of just a few individual o-MWNTs. 


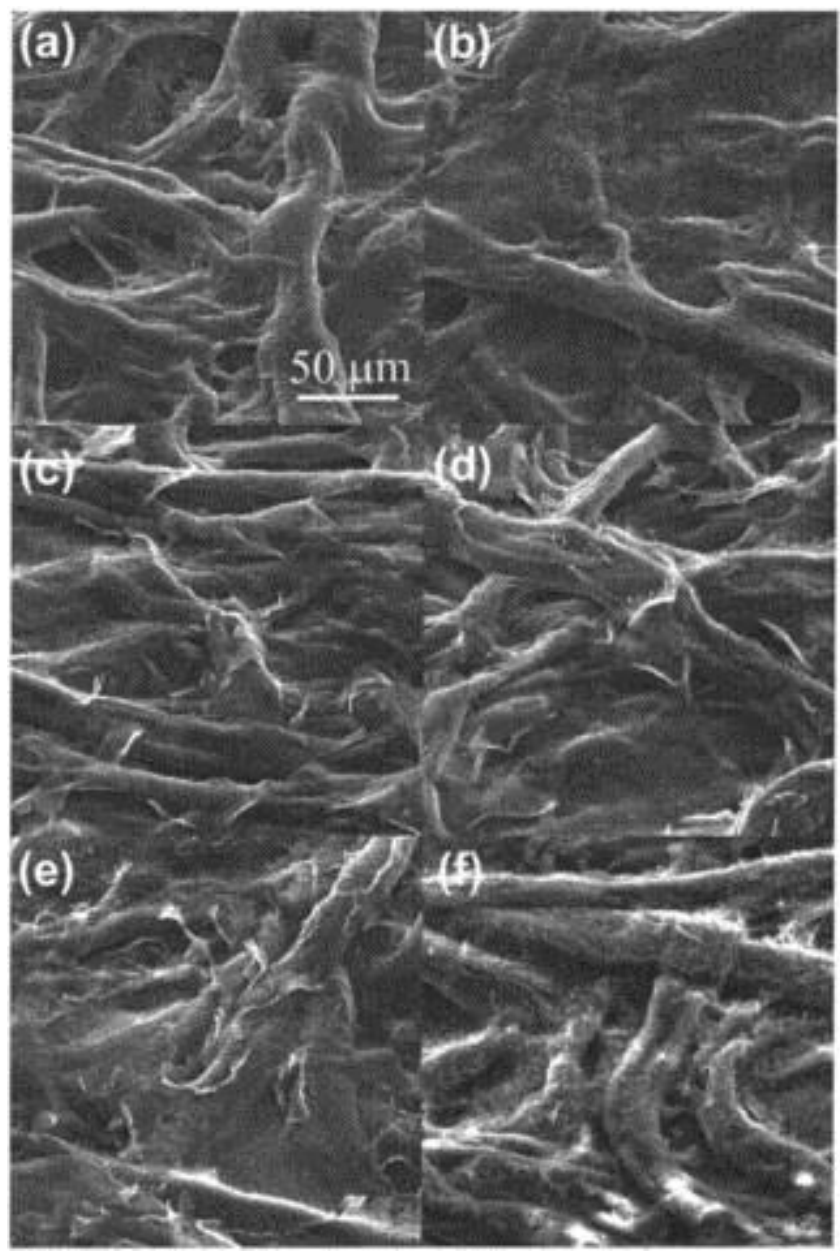

Fig 1.

[(a)-(f)] SEM images of the paper substrates coated with o-MWNTs and irradiated with laser fluences of $0,28,56,83,111$, and $139 \mathrm{~mJ} / \mathrm{cm}^{2}$, respectively.

Comparing Figs. 1a,1f, it is clear that the laser irradiation process has a profound impact on the morphology of the surface of these samples. The mechanism behind the formation of the surface features in the o-MWNT layer is likely to be associated with structural defects induced by the laser irradiation process, breaking $\mathrm{C}-\mathrm{C}$ bonds. Specifically, it is probable that structural defects occur largely at the exposed surface of the o-MWNT layer. This could be a source of mechanical stress between the top surface and the underside of the o-MWNT layer, leading to structural reformation (i.e., curling) to restore equilibrium. Equally, stresses may be inherent in the o-MWNT layer after the solvent has evaporated. Once the laser energy fractures the o-MWNT layer, these internal stresses would act to curl the o-MWNT layer adjacent to the fracture points. These scenarios fit well with the features observed in the SEM images, many of which have a uniform radius of curvature over a distance of several microns, indicative of the uniformity of defect formation.

The FE properties of the paper substrates were investigated at a vacuum better than $10^{-6}$ mbar in a simple diode configuration, with a $5 \mathrm{~mm}$ diameter spherical stainless-steel anode. Electrical connections were made via top contacts of silver paste, at the edges of the paper substrate. The 
macroscopic electric field was calculated by dividing the applied voltage by the electrode gap, typically $200 \mu \mathrm{m}$. The threshold field $\left(E_{\mathrm{th}}\right)$ was defined as the macroscopic electric field at which an emission current of $1 \mathrm{nA}$ was detected. The average $E_{\mathrm{th}}$ was calculated over six widely spaced emission sites on each sample, with the voltage cycled up and down five times at each site. The hysteresis is defined as the difference in electric field between the upward current sweep and the downward sweep. The FE characteristics of the laser irradiated substrates are presented in Fig. 2. Figure 2a shows the current-field curves and Fig. 2b shows the data plotted in Fowler-Nordheim coordinates. A large range in $E_{\mathrm{th}}$ and the slope gradients are observed, indicating that laser irradiation has a large impact on the FE properties of the o-MWNT layer.
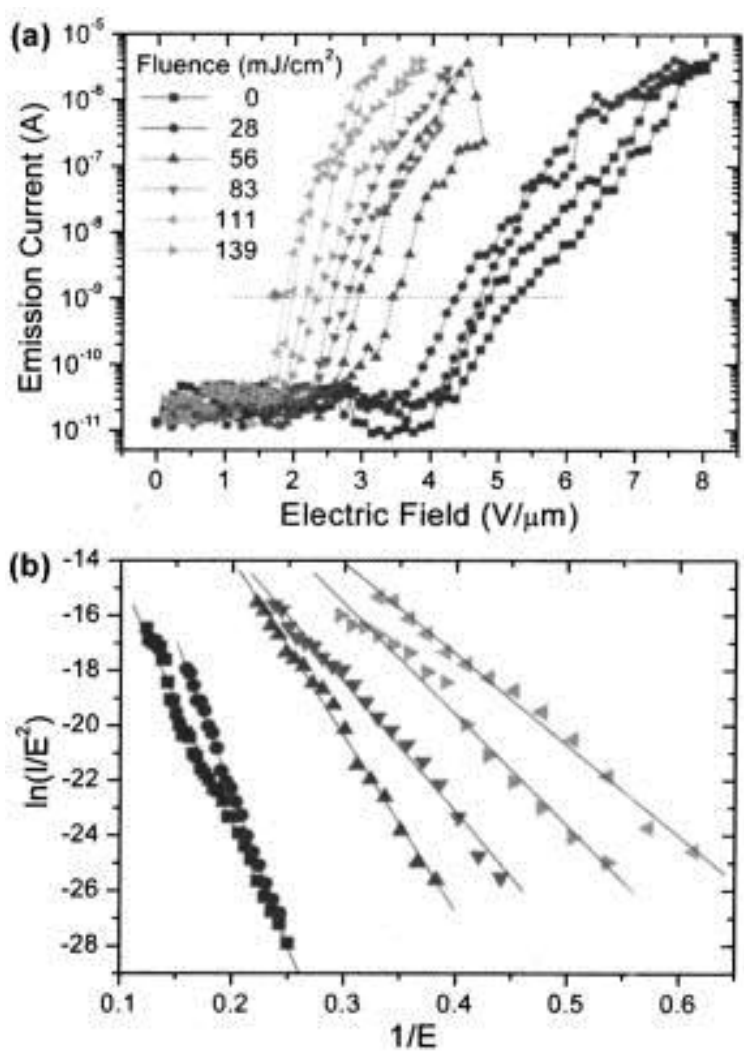

Fig 2.

(a) Field emission current-field curves for substrates subjected to various incident laser fluences. (b) Current-field data plotted in Fowler-Nordheim coordinates.

A numerical summary of the FE characteristics is presented in Table 1, while $E_{\text {th }}$ and $\beta$ are plotted against the incident laser fluence in Fig. 3. The error bars represent the standard deviation in data between the six different emission sites. Comparing untreated substrates with those irradiated at $28 \mathrm{~mJ} / \mathrm{cm}^{2}$, it is observed that both have almost identical FE characteristics, with an $E_{\text {th }}$ of 4.8 and $4.7 \mathrm{~V} / \mu \mathrm{m}$, respectively, and both having a hysteresis of $\sim 0.9 \mathrm{~V} / \mu \mathrm{m}$. This is further evidence that a laser fluence of $28 \mathrm{~mJ} / \mathrm{cm}^{2}$ has insufficient energy to restructure the o-MWNT layer. As the laser fluence increases, $E_{\mathrm{th}}$ decreases, the hysteresis decreases and $\beta$ increases. At a fluence of $111 \mathrm{~mJ} / \mathrm{cm}^{2}, E_{\mathrm{th}}$ peaks at $2.1 \mathrm{~V} / \mu \mathrm{m}$ and $\beta$ at 2307 . Also, the standard deviation is relatively low, 
suggesting better uniformity across the irradiated area. At the highest laser fluence $\left(139 \mathrm{~mJ} / \mathrm{cm}^{2}\right)$, a slight deterioration in $E_{\mathrm{th}}$ is observed, with a higher error, and $\beta$ is reduced to a value of 1895 .

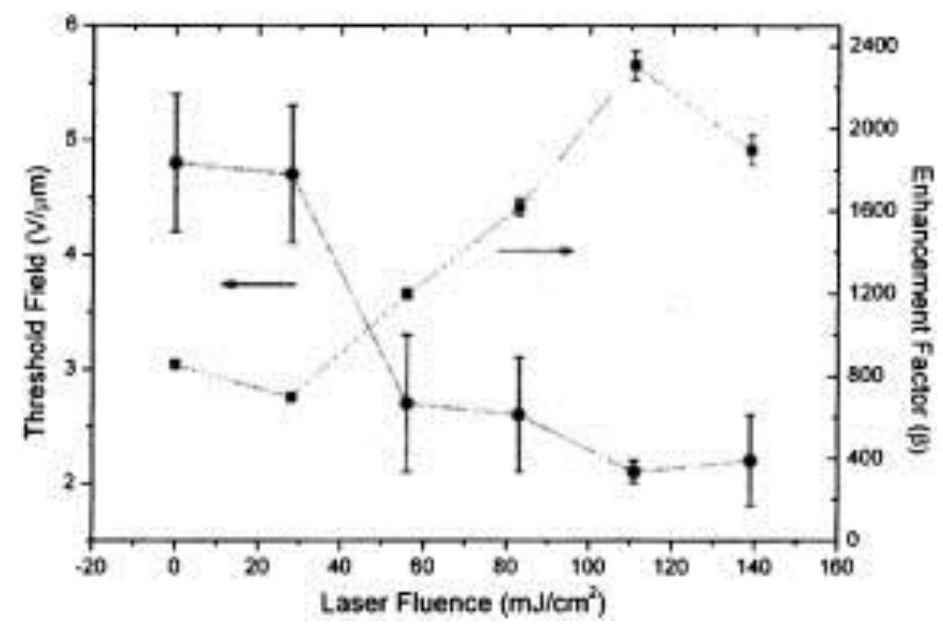

Fig 3.

$E_{\mathrm{th}}$ and $\beta$ for samples subjected to various incident laser fluences.

This improvement in the FE properties with increasing laser fluence is due to the restructuring of the o-MWNT layer by the incident laser energy, observable in the SEM images in Fig. 1. The protruding o-MWNT layers are extremely thin and would therefore be expected to have a high electric field concentration at their tips, contributing to high $\beta$. As the laser fluence increases and more protruding o-MWNT layers are created, more FE sites are created and the emission characteristics are improved. Additionally, the removal of amorphous carbon or other contaminants from the o-MWNT surface may contribute to an improvement in the FE properties. ${ }^{21,22}$ At the highest laser fluence, the FE characteristics deteriorate slightly due to extensive surface damage of the sample, leading to lower o-MWNT coverage and therefore fewer FE sites and a lower current at a given electric field. These results show strikingly similar changes in the FE characteristics to those observed by Hosono et al. ${ }^{23,24}$ In their work, a CNT-resin paste, screen printed onto glass substrates was laser treated. Here, pure o-MWNT inks on paper substrates are irradiated, suggesting that this technique can routinely be applied to CNT inks to improve their FE characteristics. Differences in the optimum laser fluence between the two studies can be explained by the difference in nature of the CNT inks and in the underlying substrates.

In summary, filter paper was used as a substrate for the deposition of MWNT ink. Subsequently, the samples were subject to laser irradiation at varying fluence. The effects of this laser irradiation process on the morphology and the FE characteristics of the samples were investigated. The morphology of the MWNT layer was dramatically altered by the laser irradiation, resulting in micronscale protrusions of the MWNT layer above the underlying substrate. This change in morphology 
contributed to an increase in $\beta$ and, therefore, $E_{\text {th }}$ was observed to improve from 4.8 to $2.1 \mathrm{~V} / \mu \mathrm{m}$, with an associated reduction in error and hysteresis. This work shows how relatively straightforward it is to tailor FE characteristics using relatively simple fabrication techniques and straightforward postdeposition treatments. This method provides a low cost, low temperature, and scalable route to manufacture efficient field emitters via ink-jet printing, dip coating, or screen printing onto readily available, flexible, large area substrates.

\section{ACKNOWLEDGMENT}

The authors would like to thank EPSRC (U.K.) for funding this research via the CBE and Portfolio Partnership awards.

\section{Tables}

Table I. Summary of numerical results.

\begin{tabular}{cccc}
\hline $\begin{array}{c}\text { Laser fluence } \\
\left(\mathbf{m} \mathbf{J} / \mathbf{c m}^{2}\right)\end{array}$ & $\begin{array}{c}\text { Threshold field } \\
(\mathbf{V} / \mu \mathbf{m})\end{array}$ & $\begin{array}{c}\text { Hysteresis at } \\
\mathbf{1} \mathbf{~ n A} \\
(\mathbf{V} / \mu \mathbf{m})\end{array}$ & $\begin{array}{c}\text { Enhancement factor } \\
(\boldsymbol{\beta})\end{array}$ \\
\hline 0 & $4.8 \pm 0.6$ & 0.9 & $854 \pm 16$ \\
$28 \pm 3$ & $4.7 \pm 0.6$ & 0.9 & $697 \pm 9$ \\
$56 \pm 6$ & $2.7 \pm 0.6$ & 0.4 & $1197 \pm 24$ \\
$83 \pm 8$ & $2.6 \pm 0.5$ & 0.4 & $1618 \pm 41$ \\
& & & $2307 \pm 70$ \\
$111 \pm 11$ & $2.1 \pm 0.1$ & 0.3 & $1895 \pm 71$ \\
\hline $139 \pm 14$ & $2.2 \pm 0.4$ & 0.3 & \\
\hline
\end{tabular}

References (24)

1. W. A. Deheer, A. Chatelain, and D. Ugarte, Science 270, 1179 (1995).

2. A. G. Rinzler et al., Science 269, 1550 (1995).

3. P. C. P. Watts, S. M. Lyth, E. Mendoza, and S. R. P. Silva, Appl. Phys. Lett. 89, 103113 (2006).

4. R. H. Baughman, A. A. Zakhidov, and W. A. Deheer, Science 297, 787 (2002). 
5. J. M. Bonard, T. Stockli, F. Maier, W. A. de Heer, A. Chatelain, J. P. Salvetat, and L. Forro, Phys. Rev. Lett. 81, 1441 (1998).

6. Q. H. Wang, A. A. Setlur, J. M. Lauerhaas, J. Y. Dai, E. W. Seelig, and R. P. H. Chang, Appl. Phys. Lett. 72, 2912 (1998).

7. S. M. Lyth, R. A. Hatton, and S. R. P. Silva, Appl. Phys. Lett. 90, 013120 (2007). [ISI]

8. J. Wang and M. Musameh, Analyst (Cambridge, U.K.) 129, 1 (2004).

9. H. Y. Shin, W. S. Chung, K. H. Kim, Y. R. Cho, and B. C. Shin, J. Vac. Sci. Technol. B 23, 2369 (2005).

10. A. P. Burden et al., J. Vac. Sci. Technol. B 18, 900 (2000).

11. H. Ago, T. Kugler, F. Cacialli, W. R. Salaneck, M. S. P. Shaffer, A. H. Windle, and R. H. Friend, J. Phys. Chem. B 103, 8116 (1999). [Inspec] [ISI] [CAS]

12. Z. Fan, T. Wei, G. Luo, and F. Wei, J. Mater. Sci. 40, 2461 (2005).

13. K. Kordas et al., Small 2, 1021 (2006).

14. L. Nilsson et al., Appl. Phys. Lett. 76, 2071 (2000).

15. R. C. Smith, R. D. Forrest, J. D. Carey, W. K. Hsu, and S. R. P. Silva, Appl. Phys. Lett. 87, 013111 (2005).

16. S. M. Lyth and S. R. P. Silva, Appl. Phys. Lett. 90, 173124 (2007).

17. A. Sawada, M. Iriguchi, W. J. Zhao, C. Ochiai, and M. Takai, J. Vac. Sci. Technol. B 21, 362 (2003).

18. P. D. Kichambare, L. C. Chen, C. T. Wang, K. J. Ma, C. T. Wu, and K. H. Chen, Mater. Chem. Phys. 72, 218 (2001).

19. Y. Zhang, T. Gong, J. Wei, W. Liu, K. Wang, and D. Wu, J. Phys. Chem. C 111, 2901 (2007).

20. J. S. Kim, K. S. Ahn, C. O. Kim, and J. P. Hong, Appl. Phys. Lett. 82, 1607 (2003

21. X. Bai, D. Li, D. Du, H. Zhang, L. Chen, and J. Liang, Carbon 42, 2125 (2004).

22. A. Hosono, T. Shiroishi, K. Nishimura, F. Abe, Z. Shen, S. Nakata, and S. Okuda, J. Vac. Sci. Technol. B 24, 1423 (2006).

23. T. Shiroishi, A. Hosono, A. Sono, K. Nishimura, Y. Suzuki, S. Nakata, and S. Okuda, J. Vac. Sci. Technol. B 24, 979 (2006). 